\title{
PERFIL DOS USUÁRIOS DE SERVIÇOS DE REABILITAÇÃO NO SISTEMA ÚNICO DE SAÚDE
}

Tainá Ribas MELO ${ }^{1}$

Luciana Vieira Castilho WEINERT ${ }^{2}$

Melissa Sayuri HOSHINO ${ }^{3}$

Tainara Piontkoski MALDANER ${ }^{4}$

\begin{abstract}
${ }^{1}$ Mestre em Educação Física - Comportamento Motor (UFPR). Bacharel em Fisioterapia (UNIOESTE). Doutoranda em Educação Física (UFPR). Fisioterapeuta da Prefeitura Municipal de Paranaguá - Paraná. tainamelo@gmail.com ${ }^{2}$ Doutora em Ciências - Engenharia Biomédica (UTFPR). Bacharel em Fisioterapia (PUC-PR). Docente do Programa de Mestrado em Desenvolvimento Territorial Sustentável e do Curso de Licenciatura em Educação Física, Setor Litoral, Universidade Federal do Paraná, Matinhos. lucianaweinert@gmail.com

${ }^{3}$ Bacharel em Fisioterapia (UNIOESTE). Fisioterapeuta da Prefeitura Municipal de Paranaguá - Paraná. melissahoshino@gmail.com

${ }^{4}$ Mestranda no Programa de Pós-Graduação em Desenvolvimento Territorial Sustentável pela Universidade Federal do Paraná. Possui graduação em Fisioterapia pela Universidade Federal do Paraná (2015). tainara.fisioufpr@ gmail.com
\end{abstract}

Recebido em: 29/08/2016- Aprovado em: 13/08/2017 - Disponibilizado em: 30/12/2017

\section{RESUMO:}

A organização do Sistema Único de Saúde (SUS) desencadeia um processo de discussão sobre os modelos assistenciais adotados e o planejamento de ações que minimizem a necessidade de reabilitação pela formulação de estratégias de promoção da saúde específicas às características que levaram ao adoecimento da população. Este estudo se justifica na medida em que o direcionamento da atuação profissional e das políticas públicas deve se pautar no conhecimento do perfil dos usuários dos serviços de saúde. Considerando-se este contexto o objetivo desta pesquisa foi analisar a utilização da fisioterapia e verificar a prevalência das patologias relacionadas aos encaminhamentos à reabilitação. $\mathrm{O}$ estudo realizado caracteriza-se como analítico observacional transversal e incluiu 1473 usuários de fisioterapia no município de Paranaguá, Paraná, Brasil entre 2006 e 2013. Os dados foram analisados descritivamente e pelos testes estatísticos de qui-quadrado e de diferença entre 2 proporções. A idade média foi de 43 anos, $56.45 \%$ do gênero feminino, com maior concentração na faixa etária adulta (13 a 59 anos). Considerando-se o comprometimento funcional e a independência, a maioria possuía afecções de baixa complexidade. Observou-se que aproximadamente $70 \%$ demandaram atendimento na área musculoesquelética, $15 \%$ na neurológica e $13 \%$ na pneumológica. As causas de encaminhamento mais prevalentes foram artrose $(11,8 \%)$, fraturas $(11,7 \%)$, acidente vascular encefálico $(7,5 \%)$ e lombalgia (6,2\%). Concluiu-se a necessidade de investimento em políticas públicas para redução das demandas de reabilitação e patologias graves.

Palavras-chave: Promoção da saúde. Reabilitação. Fisioterapia. Saúde pública. Atenção à Saúde.

\begin{abstract}
:
The organization of the Unified Health System (SUS) triggers a process of discussion on the adopted care models and planning actions that minimize the need for rehabilitation through the formulation of health promotion strategies specifics to the characteristics that led to the illness. This study is justified because the professional practice and public policy may be based on the profile of users of health. Considering this context, the aim of this research was to analyze the use of physiotherapy and determine the prevalence of diseases related to referrals to rehabilitation. We realized a cross-sectional observational analytical study including 1473 records of individuals that used physiotherapy in the city of Paranaguá, Paraná, Brazil between 2006 and 2013. The data were analyzed through descriptive statistics, chi-square and the difference between two proportions tests. The average age was 43 years, $56.45 \%$ female, with the highest concentration in the adult age group (13-59 years). Considering the functional commitment and independence, the majority had low complexity conditions. We observed that approximately $70 \%$ required care in the musculoskeletal area, $15 \%$ in neurological and $13 \%$ in respiratory. The most prevalent referring causes were arthrosis $(11,8 \%)$, fractures $(11,7 \%)$, stroke $(7,5 \%)$ and low back pain $(6,2 \%)$. We concluded the need of investment in public policies to reduce the demands of rehabilitation and serious diseases.
\end{abstract}

Keywords: Health promotion. Rehabilitation. Physical therapy specialty. Public health. Health care. 


\section{INTRODUÇÃO}

A implantação do Sistema Único de Saúde (SUS) tem suscitado reflexões acerca dos modelos assistenciais adotados, bem como das práticas profissionais. A necessidade de adequação das profissões à realidade epidemiológica e à nova lógica de organização dos sistemas de serviços de saúde é emergente (BISPO JÚNIOR, 2010). A Atenção Primária em Saúde (APS) constitui uma base estruturante deste Sistema (NEVES e ACOLE, 2011), caracterizando um espaço onde ocorre o primeiro contato com o paciente, onde existe capacidade para a resolução de grande parte dos problemas de saúde por eles apresentados (LAVRAS, 2011). Neste nível de atenção realiza-se, prioritariamente, ações de prevenção e promoção de saúde (BRASIL, 2012).

Nos últimos anos, o conceito de promoção da saúde tornou-se um marco para as políticas de saúde em nível mundial (BOTTI e SCOCHI, 2006). O paradigma da promoção da saúde apresentado pela Carta de Ottawa é o de que a saúde deve ser entendida em seu conceito amplo, ou seja, os indivíduos e famílias devem ser assistidos antes do surgimento dos problemas e agravos de sua saúde (DELAI e WISNIEWSKI, 2011).

Para se adequar a este novo paradigma a atuação profissional do fisioterapeuta tem evoluído historicamente. A fisioterapia surgiu no Brasil em um contexto de reabilitação para atender as sequelas de Poliomielite nos anos
50. Na década de 70 a atuação se expandiu para uma ação curativa em outras áreas. Somente após a reforma sanitária de 1980 e a implantação do SUS, ocorreu gradualmente a inserção profissional nos diferentes segmentos de atenção à saúde, que evoluiu com a sua progressiva participação nas equipes de atenção primária em saúde (SÁ e GOMES, 2013). Assim, o fisioterapeuta insere-se no sistema de atenção primária numa nova perspectiva de atuação, focada nas práticas de prevenção e promoção e não restrita aos procedimentos de reabilitação. É preciso voltar os olhos para uma físioterapia de controle de riscos, e não de controle de danos (BISPO JÚNIOR, 2010). E, para compreender o caminho que o fisioterapeuta deve realizar para direcionar suas ações prioritariamente à promoção de saúde e secundariamente à reabilitação é importante conhecer o perfil dos usuários dos serviços de reabilitação.

A busca nas bases de dados LILACS (Literatura Latino-Americana e do Caribe em Ciências da Saúde), Medline (Medical Literature Analysis and Retrieval System Online) e SciELO (Scientific Eletronic Library Online) por meio do descritor "fisioterapia" associado aos termos "epidemiologia", "SUS", "serviço público", "PSF" e "unidades sanitárias", reportou apenas quatro estudos sobre o uso da fisioterapia, dois conduzidos em Pelotas, Rio Grande do Sul (SIQUEIRA, FACCHINI e 
HALLAL， 2005; VASCONCELOS, FAGUNDES e GIUSTI, 2007), um conduzido em Lages, Santa Catarina (MORETTO et al., 2009) e, outro na Colômbia (BAQUERO e LÓPEZ, 2004). Os demais artigos encontrados referiam-se à utilização da fisioterapia no tratamento e na reabilitação de pacientes ou abordaram a formação acadêmica do profissional.

Portanto, são poucos os trabalhos que definem o perfil dos usuários da reabilitação, e são ainda mais escassos os estudos com relação às especialidades e patologias mais prevalentes nesta população. Tal constatação é relevante por indicar a necessidade de novas pesquisas que descrevam a prevalência do uso de serviços de fisioterapia no Brasil e que testem sua distribuição segundo características específicas dos indivíduos. Entende-se que tais informações possibilitam a identificação desta população e o planejamento de ações que minimizem a necessidade de reabilitação pela formulação de estratégias de promoção da saúde específicas às características que levaram ao adoecimento da população.

Desta maneira, o presente estudo pretende contribuir preenchendo parcialmente esta lacuna, ao analisar a prevalência das patologias que levam a busca por reabilitação nas diferentes áreas da fisioterapia, com o objetivo de embasar e orientar ações de promoção da saúde que podem ser efetuadas na APS para diminuir as demandas por ações curativas nos demais níveis de atenção.

\section{METODOLOGIA}

Esta pesquisa foi um estudo analítico observacional transversal (MARQUES e PECCIN, 2005), aprovado pelo Comitê de Ética em Pesquisa do Setor de Ciências da Saúde da Universidade Federal do Paraná (UFPR) (CAAE: 13314813.5.0000.0102), que ocorreu no município de Paranaguá, Paraná. Situado na região litorânea do estado, a 100 km da capital, sua população em 2010 era de 140.469, e seu Índice de Desenvolvimento Humano Municipal igual a 0,750 (IBGE 2014).

Foram analisados os indivíduos atendidos em reabilitação no período de 2006 a 2013, totalizando 1473 registros. Elaborouse um questionário estruturado com base nas informações disponíveis contendo questões fechadas e categóricas, que abrangeram as variáveis: bairro de residência, patologia e/ou motivo de encaminhamento (descritivo), código da CID10 (Classificação Estatística Internacional de Doenças e Problemas Relacionados com a Saúde) e área da fisioterapia em que foram realizados os atendimentos (musculoesquelética, neurologia, pneumologia, cardiovascular, oncologia, ginecologia e obstetrícia, e, dermatofuncional).

Também foram incluídas informações registradas pelo fisioterapeuta quanto ao nível 
de comprometimento do estado de saúde e independência nas atividades de vida diárias. Nesta questão o paciente poderia ser classificado em baixa, média ou alta complexidade.

Para diagnosticar o perfil demográfico da população foram considerados os dados de sexo e idade. A idade foi dividida em três faixas etárias: 0 a 12 anos (infanto-juvenil), 13 a 59 anos (adulto), 60 anos ou mais (idosos).

Os dados foram analisados descritivamente e por testes estatísticos como o teste de Qui-quadrado e o teste de diferença entre 2 proporções.

\begin{tabular}{|c|c|c|c|c|}
\hline Gênero & $\mathrm{N}$ & $\%$ & Idade(anos) & DV \\
\hline Feminino & 831 & $56,45^{*}$ & $47,22 *$ & $\pm 21,59$ \\
\hline Masculino & 641 & 43,55 & 38,17 & $\pm 22,83$ \\
\hline Total & 1473 & $\mathrm{p}<0,001$ & $\mathrm{p}<0,001$ & \\
\hline
\end{tabular}

Observa-se pela Tabela 2 que a especialidade de maior demanda é a área de musculoesquelética (alterações osteomioarticulares) relacionada à traumatologia, ortopedia e reumatologia (70,47\%) seguida pelas áreas de neurologia indivíduos que procuraram pelo serviço de fisioterapia foi de 43 anos, com mínimo de 0,1 e máximo de 95, com desvio-padrão de 22,58. Desses, $56.45 \%$ eram do gênero feminino e 43,55\% masculino. Comprovou-se estatisticamente a significância de tal diferença na distribuição da amostra por meio do teste de Qui-quadrado $(\mathrm{p}<0,001)$. Pelo teste de diferença entre 2 proporções, as mulheres utilizaram mais o serviço de fisioterapia. Considerando-se a idade em cada gênero também se observa diferença $(\mathrm{p}<0,001)$ na idade média dos sujeitos e as mulheres geralmente têm idade média $(47,22 \pm 21,59$ anos $)$ superior a dos homens $(38,17 \pm 22,83$ anos $)$, como se observa na Tabela 1.

\section{RESULTADOS}

Durante a análise dos prontuários pode-se observar que a média de idade dos

Tabela 1 - Caracterização da amostra quanto a gênero e idade

Fonte: os autores.

$(15,41 \%)$ e pneumologia $(13,10 \%)$. Áreas como cardiologia e oncologia tiveram uma prevalência baixa de 0,34 e $0,68 \%$ respectivamente, e outras como ginecologia e dermatofuncional não tiveram registros de procura. 
Tabela 2 - Número de pacientes por especialidade, nível de complexidade e faixa etária

\begin{tabular}{lccccccccccc}
\hline & \multicolumn{3}{c}{$\begin{array}{c}\text { Infanto-Juvenil } \\
\text { (0MÊS-12 ANOS) }\end{array}$} & \multicolumn{3}{c}{$\begin{array}{c}\text { Adolescente/ Adulto } \\
(13-59 \text { ANOS })\end{array}$} & \multicolumn{3}{c}{$\begin{array}{c}\text { Idoso } \\
\text { (> 60 ANOS) }\end{array}$} & Total & $\%$ \\
\hline ÁREA & Baixa & Média & Alta & Baixa & Média & Alta & Baixa & Média & Alta & \\
\hline ME & 71 & 6 & 0 & 676 & 33 & 2 & 222 & 27 & 1 & 1038 & 70,47 \\
Neuro & 4 & 28 & 1 & 27 & 78 & 4 & 8 & 77 & 0 & 227 & 15,41 \\
Pneumo & 113 & 7 & 0 & 24 & 15 & 0 & 5 & 27 & 2 & 193 & 13,10 \\
Cardio & 0 & 0 & 0 & 1 & 1 & 2 & 1 & 0 & 0 & 10 & 0,34 \\
Onco & 0 & 0 & 0 & 1 & 4 & 0 & 0 & 5 & 0 & 10 & 0,68 \\
GO & 0 & 0 & 0 & 0 & 0 & 0 & 0 & 0 & 0 & 0 & 0,00 \\
Dermato & 0 & 0 & 0 & 0 & 0 & 0 & 0 & 0 & 0 & 0 & 0,00 \\
Total & 188 & 41 & 1 & 729 & 131 & 8 & 236 & 136 & 3 & 1473 & 100,0 \\
\hline
\end{tabular}

$\mathrm{ME}=$ musculoesquelética, Neuro $=$ neurologia, Pneumo $=$ pneumologia, Cardio $=$ cardiovascular, Onco $=$ oncologia, $\mathrm{GO}=$ ginecologia e obstetrícia, Dermato= dermatofuncional.

Baixa/ Média/Alta= níveis de complexidade (comprometimento funcional)

Fonte: os autores.

Percebe-se que a faixa etária que representa a maior procura por fisioterapia está entre 13 e 59 anos de idade, pois mais de $50 \%$ da amostra foi composta por adolescentes e adultos jovens, cujos níveis de comprometimento funcional corresponderam principalmente à baixa e média complexidade (Tabela 2). Quanto a estes níveis, o mesmo ocorreu nas demais faixas etárias, de modo que entre os idosos e os mais jovens se observa maior número de atendimentos de baixa complexidade, seguido da média complexidade.

Entre todos os prontuários pesquisados apenas $160(11,34 \%)$ foram encaminhados com código da CID10. Ainda, 273 (18,54\%) pacientes foram encaminhados à fisioterapia sem qualquer informação adicional, ou seja, sem código da CID10, sem diagnóstico clínico descritivo e sem sinais e sintomas que pudessem caracterizar o motivo do encaminhamento. Os demais $(70,12 \%)$ foram encaminhados com base em sinais e sintomas (por exemplo, lombalgia) ou área de especialidade (por exemplo, fisioterapia motora e fisioterapia respiratória). Desta forma, durante a triagem no serviço de fisioterapia, para alguns pacientes definiu-se somente a área de especialidade da fisioterapia em que o mesmo receberia atendimento, e não o diagnóstico devido à escassez de informações clínicas.

Os prontuários com motivo de encaminhamento conhecido foram agrupados em 113 categorias, segundo a patologia. Quando não foi possível identificar a patologia caracterizou-se a lesão ou o sinal ou o sintoma que originaram a demanda pelo serviço de fisioterapia. Apenas nos acometimentos do sistema respiratório, não houve descrição do diagnóstico, ou identificação da patologia e de sinais e sintomas que justificassem a procura pela fisioterapia. A Tabela 3 apresenta uma análise de proporção em que se aferem os motivos de encaminhamento mais prevalentes, 
independente da área de especialidade da

fisioterapia.

Tabela 3. Prevalência das causas do encaminhamento para o serviço de fisioterapia

\begin{tabular}{lc}
\hline \multicolumn{1}{c}{ Patologia } & \% de pacientes atendidos \\
\hline Sistema respiratório (sem diagnóstico) & 25,0 \\
Artrose & 11,8 \\
Fraturas & 11,7 \\
Acidente Vascular Encefálico (AVE) & 7,5 \\
Lombalgia & 6,2 \\
Escoliose & 2,4 \\
Tendinite & 2,1 \\
Doença Pulmonar Obstrutiva Crônica (DPOC) & 2,0 \\
Lombociatalgia & 2,0 \\
Paralisia facial & 1,8 \\
Hérnia discal & 1,7 \\
Bronquite & 1,5 \\
Esporão de calcâneo & 1,4 \\
Paralisia cerebral & 1,0 \\
Dorsalgia & 1,0 \\
Entorse de tornozelo & 1,0 \\
Cervicalgia & 0,8 \\
Asma & 0,7 \\
Atraso no Desenvolvimento Neuropsicomotor (DNPM) & 0,7 \\
Outros & 17,6 \\
\hline
\end{tabular}

Fonte: os autores.

Observa-se que as maiores demandas são as do sistema respiratório, seguidas por artrose e fraturas (agrupadas independente do local de lesão), Acidente Vascular Encefálico (AVE) e lombalgia. Os encaminhamentos de menor ocorrência com valores inferiores a $0,5 \%$ e que foram citados apenas uma vez foram agrupados com denominação "outros".

Nesta análise, o teste de Qui-quadrado indicou que a motivação para o encaminhamento é influenciada pela variável gênero $(p=0,0)$. O teste de diferença entre 2 proporções $(\mathrm{p}<0,05)$ indicou algumas ocorrências mais frequentes no gênero feminino, como artrose $(74 \%)$, escoliose (77,1\%), DPOC (79,3\%), esporão de calcâneo $(90,5 \%)$, cervicalgia $(91,7 \%)$, bursite $(100 \%)$, e dorsalgia (100\%). Enquanto outras são maiores no gênero masculino, como as demandas do sistema respiratório $(51,4 \%)$, atraso no DNPM (80\%), hérnia discal (72\%), menisectomia $(100 \%)$, osteomielite $(100 \%)$, pós-operatório de lesão do ligamento cruzado anterior (100\%) e tenorrafias (100\%). As causas mais prevalentes de encaminhamento, como fraturas, AVE e lombalgia, não apresentaram diferença de frequência entre os gêneros. 


\section{DISCUSSÃO}

O reconhecimento do uso e das necessidades da população em relação à fisioterapia contribui para a formação de subsistemas de informações com a finalidade de avaliação de ações e estratégias na área, e também para a vigilância e o planejamento de políticas em saúde (BAQUERO E LÓPEZ, 2004; SILVA, LIMA e LEROY, 2013). Apesar de a fisioterapia ser uma profissão regulamentada há mais de 4 décadas, a oferta de atendimento pelo SUS ainda é pequena quando se considera a demanda existente (BIM, PELLOSO e PREVIDELLI, 2011; CASTRO, NEVES e ACIOLE, 2011).

A média de idade dos indivíduos desta pesquisa que fizeram fisioterapia, em ambos os gêneros, foi de 43 anos, com desvio-padrão de 22,58. O estudo de Siqueira, Facchini e Hallal (2005) apresentou média semelhante, de 43,2 anos, e também pesquisou a demanda por fisioterapia. Pesquisa realizada sobre fatores associados à utilização de serviços ambulatoriais entre homens e mulheres também obteve dados similares, com idade média de 40,3 anos (MENDOZA-SASSI, BÉRIA e BARROS, 2003). Em contrapartida, o estudo de Ferrer e colaboradores (2015) apresentou média de idade de pacientes da lista de espera de atendimento aos serviços de fisioterapia de 54,96 anos, com desvio padrão de 17,04 anos. Com o passar dos anos, a saúde necessita de maiores cuidados, o que justifica a associação do uso da fisioterapia com a idade (BIM, PELLOSO e PREVIDELLI, 2011).

No gênero masculino, a idade média foi de 38,17 anos com desvio-padrão de 22,83 . Com relação à faixa etária os estudos existentes no Brasil relatam a idade média dos participantes, porém sem distinção de gênero (MORETTO et al., 2009; SIQUEIRA, FACCHINI e HALLAL 2005).

No gênero feminino, a idade média foi de 47 anos com desvio-padrão de 21,59. Bim, Pelloso e Previdelli (2011) realizaram um inquérito domiciliar sobre o uso de fisioterapia entre mulheres e verificaram idade média de 41 anos, com desvio-padrão de 14,93. Além disto, verificaram que quanto mais idade tiver a mulher, maior será a chance de ela usar os serviços da fisioterapia, com uma taxa de acréscimo de 0,033 por ano de idade. No presente estudo, a idade média foi um pouco superior $(14,63 \%)$ e o desviopadrão foi bastante superior $(44,61 \%)$. Isto ocorreu devido à influência da idade mínima que neste estudo foi de 0,1 anos e no primeiro de 18 anos.

Observou-se que as mulheres receberam mais encaminhamentos médicos para fisioterapia $(56,45 \%)$ que os homens $(43,55 \%)$. Em estudo sobre demandas de serviços ambulatoriais médicos também houve maior demanda feminina $(53,9 \%)$ (MENDOZA-SASSI, BÉRIA e BARROS, 2003), assim como no estudo de Silva e Sirena (2015) que traçou o perfil de 
encaminhamentos a fisioterapia no Rio Grande do Sul (70,5\%), e no estudo de Ferrer e colaboradores (2015) que verificou a lista de espera de atendimento aos serviços de fisioterapia $(56,96 \%)$. Tal fato pode ser explicado pelo aumento da longevidade das mulheres, assim como o direcionamento de políticas públicas especificas para esta população (PINHEIRO et al., 2002).

Contrariamente, os estudos de Siqueira, Facchini e Hallal (2005) e Moretto e colaboradores (2009) apresentaram ligeira tendência a maior utilização dos serviços de fisioterapia pelos homens. A diferença nos resultados pode ser porque nos estudos em que houve maior procura pelos homens não se analisou exclusivamente pacientes com encaminhamento médico. No presente estudo todos os usuários de fisioterapia que compuseram a amostra tinham obrigatoriamente encaminhamento médico. Estudos apontam para o fato de homens cuidarem menos da saúde que as mulheres desde o nível de atenção primária até situações de maior complexidade e risco de morbidade (GOMES, NASCIMENTO e ARAUJO, 2007). Figueiredo (2005) reforça que existe uma ideia equivocada e disseminada de que os serviços de saúde são destinados a mulheres, crianças e idosos pela questão da identidade masculina que não se vê como necessitando desses cuidados. Assim, o uso dos serviços de saúde pelos homens difere daquele feito pelas mulheres, concentrando-se na assistência a agravos e doenças, em que a busca por atendimento acontece em situações extremas de emergência ou em nível especializado ou de urgência (MOURA et al., 2014).

Os achados deste estudo também demonstram essa tendência ao evidenciar que a população que mais procura o serviço de reabilitação $(>50 \%)$ está na faixa etária entre 13 e 59 anos. Outros estudos também apontaram esta prevalência na fase adulta, bem como aumento da demanda por fisioterapia proporcionalmente ao aumento da idade (MORETTO et al., 2009; SIQUEIRA, FACCHINI e HALLAL, 2005). Nenhum dos referidos estudos incluiu usuários com faixa etária inferior a 20 anos.

O presente estudo corrobora com os achados do panorama nacional apontados por Castro, Neves e Aciole (2011) de que a área de maior atendimento é a de pacientes com desordens motoras $(61,8 \%)$. Pode-se afirmar que a tendência local segue a nacional, pois no estudo atual verificou-se maiores demandas nas especialidades musculoesquelética $(70,47 \%)$ e neurológica $(15,41 \%)$, e, ambas são as promotoras das desordens motoras.

Independente da área de especialidade da fisioterapia envolvida no tratamento do paciente, ao classificar os prontuários por patologia ou sinais e sintomas, a artrose foi a patologia de maior ocorrência $(11,8 \%)$, seguida por fraturas $(11,7 \%)$, AVE $(7,54 \%)$ e 
lombalgia $(6,2 \%)$. O fato de patologias como a artrose e a ocorrência de sintomas como a lombalgia terem alta demanda de atendimento reafirma as tendências globais de adoecimento da população (COIMBRA et al., 2004; LIPOSCKI e ROSA NETO, 2008; TOSCANO e EGYPTO, 2001).

Estudos americanos apontam que mais de 50 milhões de pessoas apresentam artrose no mundo ${ }^{18}$. Estudos recentes realizados no Brasil tiveram resultados semelhantes, com a osteoartrose que ocupa o topo da lista de doenças atendidas nos serviços de fisioterapia (36\%) (FERRER et al., 2015) e (29\%) (SILVA E SIRENA, 2015). No presente estudo além de ser a patologia mais prevalente observou-se que a ocorrência de artrose é bem maior em mulheres (74\%) dos casos.

A dor e a dificuldade motora causada por condições como a artrose e a lombociatalgia aumentam o risco de quedas e complicações importantes como as fraturas (LIPOSCKI e ROSA NETO, 2008). Esta foi a segunda maior causa de encaminhamento para fisioterapia levantada pela presente pesquisa, o que corrobora com os resultados dos outros estudos (FERRER et al., 2015; SILVA E SIRENA, 2015).

A lombalgia é sintomatologia de elevada ocorrência em todo o mundo e estimativas apontam que pode afetar $75-80 \%$ da população em algum momento da vida (ALMEIDA et a., 2008; TOSCANO e
EGYPYO, 2001). Esta foi uma queixa elevada no presente estudo, cujos índices podem ser reduzidos com investimentos em educação em saúde. O enfoque em prevenção no posto de trabalho e realização de atividades físicas regulares podem diminuir os fatores de risco (ALMEIDA et a., 2008).

O elevado número de indivíduos com AVE merece destaque por ser a terceira patologia apontada como maior demanda de fisioterapia. Este resultado corrobora com estatísticas nacionais e internacionais que apontam para a gravidade desta patologia. $\mathrm{O}$ AVE é a segunda principal causa de morte no mundo (ALMEIDA, 2012), a principal causa de mortes no Brasil entre óbitos por doenças cerebrovasculares e a principal causa de incapacidade no mundo (BRASIL, 2011). Além de elevada mortalidade, a maioria dos sobreviventes apresenta sequelas, com limitação da atividade física e intelectual/cognitiva e elevado custo social para cuidados com a saúde além do afastamento do trabalho (PEREIRA et al., 2009). Isto reitera a importância de se controlar os fatores de risco vascular que tem causado aumento na incidência de casos de AVE associados à aterosclerose (YAMAMOTO, 2011).

Quanto ao nível de comprometimento do estado de saúde e independência nas atividades de vida diária, a maioria dos pacientes foi classificada com acometimentos de baixa complexidade $(78,27 \%)$, alguns de 
média complexidade $(20,91 \%)$ e poucos de alta $(0,81 \%)$. Não se encontrou estudos na área da fisioterapia que fizessem registro destas informações e para comparar os resultados com os deste estudo.

Ressalta-se a escassez de informações sobre diagnóstico, observado pelo baixo número de pacientes encaminhados a fisioterapia com código da CID10 (11,34\%) e pelo número de pacientes sem diagnóstico ou qualquer outro sinal e sintoma descrito em seu prontuário (18,54\%). Essa falta de informação prejudica a elaboração de um plano de tratamento seguro e eficaz, a integração entre profissionais de diferentes áreas e o acompanhamento sistemático do paciente (FERREIRA, POTERIKO e FACCI, 2010). Além disto, dificulta programar o tempo necessário para o atendimento deste paciente, bem como priorizar o atendimento de indivíduos com maior comprometimento funcional. Analogamente ao verificado na literatura sobre nível de comprometimento funcional dos pacientes atendidos pela fisioterapia, também não se encontrou informações sobre a presença do diagnóstico nos encaminhamentos médicos para fisioterapia.

Dados mostram que a revolução demográfica brasileira constitui uma conquista e uma responsabilidade para os gestores públicos e a sociedade. É crucial investir na promoção da autonomia e da vida saudável, assim como prover atenção adequada às necessidades específicas (MINAYO, 2012). Atualmente o SUS busca o redirecionamento do foco em ações na atenção curativa para um cuidado centrado nas necessidades de saúde, com ênfase em ações de promoção de saúde e prevenção de doenças (SATO et al., 2015).

Neste contexto esta pesquisa contribui para que a atuação do fisioterapeuta seja alicerçada em evidências de pesquisas científicas e em indicadores de saúde (MARQUES e PECCIN, 2005). O perfil das demandas atendidas é relevante para mensuração destes indicadores (CASTRO, NEVES e ACIOLE, 2011). Assim, o conhecimento e o dimensionamento das características da oferta dos serviços de saúde no país são decisivos para a fundamentação de estratégias mais específicas nas políticas públicas de saúde.

Embora a presença do fisioterapeuta na APS seja assegurada e fundamentada (BRASIL, 2008), na prática a profissão ainda percorre seus primeiros passos, na busca de espaço neste nível de atenção à saúde. Neste contexto, Ferrer e colaboradores (2015) concluíram em seus estudos que a alta demanda de pacientes na fisioterapia da Atenção Secundária pode ser explicada pela baixa resolutividade da mesma na APS, onde existe atendimento inadequado, falta de triagem e prioridade no atendimento.

Com o desenvolvimento de pesquisas acerca do perfil de determinada população, 
espera-se que os gestores de saúde tenham subsídios necessários para a consolidação do profissional fisioterapeuta na APS, através da elaboração de planos e estratégias, visando a promoção da saúde e prevenção de doenças.

Neste estudo verificaram-se informações importantes como a idade média dos pacientes atendidos em fisioterapia, a maior procura do público feminino e a ocorrência de mais demanda pelas especialidades musculoesquelética, neurologia e pneumologia. Além disto, identificou-se as informações constantes nos

\section{REFERÊNCIAS}

ALMEIDA, I.C.G.B. et al. Prevalência de dor lombar crônica na população da cidade de Salvador. Rev. bras. ortop., São Paulo, v. 43, n. 3, p. 96-102, Mar. 2008. http://dx.doi.org/10.1590/S0102$\underline{36162008000200007}$

ALMEIDA, S.R.M. Análise epidemiológica do acidente vascular cerebral no Brasil. Rev Neurocienc. v.20, n.4, p. 481-2, 2012. http://doi: 10.4181/RNC.2012.20.483ed.2p

BAQUERO, G.; LÓPEZ, O. El papel de la epidemiología en la identificación de necesidades relacionadas com la fisioterapia em Colombia. Salud Publica Mex., v.46, n.1, p.5-6, 2004. http://dx.doi.org/10.1590/S0036$\underline{36342004000100001}$

BIM, C.R.; PELLOS, O.S.M.; PREVIDELLI, I.T.S. Inquérito domiciliar sobre uso da fisioterapia por mulheres em GuarapuavaParaná-Brasil. Cien Saude Colet., v.16, n.9, p.3837-44, 2011. http://dx.doi.org/10.1590/S1413$\underline{81232011001000019}$ encaminhamentos dos pacientes, que o nível de comprometimento e funcionalidade dos pacientes se concentra prioritariamente nos atendimentos de baixa complexidade, e que quanto a prevalência as patologias e sintomas mais frequentes são artrose, fraturas, AVE e lombalgia. Como tais informações são corroboradas em outros estudos nacionais e internacionais, salienta-se a importância de se investir em estratégias de promoção da saúde para reverter os índices de prevalência de patologias graves como o AVE, e, incapacitantes como a osteoartrose.

BISPO JÚNIOR, J.P. Fisioterapia e saúde coletiva: desafios e novas responsabilidades profissionais. Cien Saude Colet., v.15, n.1, p.1627-36, 2010.

http://dx.doi.org/10.1590/S1413$\underline{81232010000700074}$

BOTTI, M.L.; SCOCHI, M.J. O aprender organizacional: relato de experiência em uma Unidade Básica de Saúde. Saude Soc., v.15, n.1, p.107-14, 2006.

http://dx.doi.org/10.1590/S0104$\underline{12902006000100011}$

BRASIL. Ministério da Saúde. Portaria ${ }^{\circ}$ 154, de 24 de janeiro de 2008. Cria os Núcleos de Apoio à Saúde da Família NASF. Diário Oficial da República Federativa do Brasil, Brasília (DF), 2008 jan 25. Seção 1:18. http://dab.saude.gov.br/docs/legislacao/portari a154_24_01_08.pdf

BRASIL. Ministério da Saúde. Política nacional de atenção básica. Brasília: Ministério da Saúde, 2012. http://189.28.128.100/dab/docs/publicacoes/g eral/pnab.pdf

BRASIL. Ministério da Saúde (MS).

Consulta Pública no 7 - Institui a Linha de 
Cuidado do Acidente Vascular Cerebral (AVC) no âmbito do Sistema Único de Saúde (SUS). Brasília: Ministério da Saúde; 2011.

http://www.cadastro.abneuro.org/site/CONSU LTA\%20PUBLIC_AVC_NOV2011.pdf

CASTRO, A.P.; NEVES, V.R.; ACIOLE, G.G. Regional differences and costs of physical therapy procedures in Brazil's unified health system, 1995 to 2008. Rev

Panam Salud Publica, v.30, n.5, p..469-76, 2011. http://dx.doi.org/10.1590/S1020$\underline{49892011001100010}$

COIMBRA, I., et al. Osteoartrite (artrose): tratamento. Rev Bras Reumatol., v.44, n.6, p.450-3, 2004. http://dx.doi.org/10.1590/S048250042004000600009

DELAI, K.D.; WISNIEWSKI, M.S.W. Inserção do fisioterapeuta no Programa Saúde da Família. Cien Saude Colet., v.16, n.1, p. 1515-23, 2011. http://dx.doi.org/10.1590/S1413$\underline{81232011000700087}$

FERREIRA, L.; POTERIKO, J.F.; FACCI, L.M. Caracterização dos encaminhamentos de pacientes traumato-ortopédicos e reumáticos a clínica de fisioterapia do Cesumar. Revista Científica JOPEF, v.1, n.1, p.106-114, 2010. http://www.cesumar.br/prppge/pesquisa/mostr as/quin_mostra/larissa_ferreira.pdf

FERRER, M.L.P., et al . Microrregulação do acesso à rede de atenção em fisioterapia: estratégias para a melhoria do fluxo de atendimento em um serviço de atenção secundária. Fisioter. Pesqui., São Paulo, v. 22, n. 3, p. 223-230, 2015 . http:/ DOI: $\underline{10.590 / 1809-2950 / 13038422032015}$

FIGUEIREDO, W. Assistência à saúde dos homens: um desafio para os serviços de atenção primária. Cien Saude Colet., v.10, n.1, p.105-9, 2005.

http://dx.doi.org/10.1590/S1413-

$\underline{81232005000100017}$
GOMES, R.; NASCIMENTO, E.F.;

ARAUJO, F.C. Por que os homens buscam menos os serviços de saúde do que as mulheres? As explicações de homens com baixa escolaridade e homens com ensino superior. Cad Saude Publica., v.23, n.3, p.565-74, 2007.

http://dx.doi.org/10.1590/S0102-

$\underline{311 X 2007000300015}$

INSTITUTO BRASILEIRO DE GEOGRAFIA E ESTATÍSTICA (IBGE).

Diretoria de pesquisas, coordenação de população e indicadores sociais [site]. Rio de Janeiro: IBGE; 2014.

http://cidades.ibge.gov.br/ xtras/perfil.php?lang $=\&$ codmun $=411820 \&$ sea $\underline{\mathrm{rch}=\text { parana|paranagua }}$

LAVRAS, C. Atenção Primária à Saúde e a Organização de Redes Regionais de Atenção à Saúde no Brasil. Saúde Soc. São Paulo, v.20, n.4, p.867-874, 2011. http://dx.doi.org/10.1590/S0104$\underline{12902011000400005}$

LIPOSCKI, D.B.; ROSA NETO, F. Prevalência de artrose, quedas e a relação com o equilíbrio de idosos. Ter Man., v.6, n.26, p.235-8, 2008.

MARQUES, A.P.; PECCIN, M.S. Pesquisa em fisioterapia: a prática baseada em evidências e modelos de estudos. Fisioter Pesq., v.12, n.1, p.43-8, 2005.

MENDOZA-SASSI R, BÉRIA JU, BARROS AJD. Fatores associados à utilização de serviços ambulatoriais: estudo de base populacional. Rev Saude Publica, v.37, n.3, p.372-8, 2003.

http://dx.doi.org/10.1590/S0034$\underline{89102003000300017}$

MINAYO, M.C.S. O envelhecimento da população brasileira e os desafios para o setor saúde. Cad Saude Publica, v.28, n.2, p.20810, 2012.

MORETTO, LC., et al. Prevalence of the use of physical therapy services among the urban 
adult population of Lages, Santa Catarina.

Braz J Phys Ther., v.13, n.2, p.130-5, 2009. http://dx.doi.org/10.1590/S1413$\underline{35552009005000023}$

MOURA, E.C., et al. Atenção à saúde dos homens no âmbito da Estratégia Saúde da Família. Cien Saude Colet., v.19, n.2, p.42938, 2014. http://dx.doi.org/10.1590/1413$\underline{81232014192.05802013}$

NEVES, L.M.T.; ACIOLE, G.G. Desafios da integralidade: revisitando as concepções sobre o papel do fisioterapeuta na equipe de Saúde da Família. Interface (Botucatu), Botucatu , v. 15, n. 37, p. 551-564, 2011.

http://www.scielo.br/scielo.php?script=sci_art text\&pid=S1414-

$\underline{32832011000200018 \& \operatorname{lng}=\mathrm{en} \& \mathrm{nrm}=\mathrm{iso}}$

PEREIRA, A.B.N.G., et al. Prevalência de acidente vascular cerebral em idosos no município de Vassouras, Rio de Janeiro, Brasil, através do rastreamento de dados do Programa Saúde da Família. Cad Saude

Publica, v.25, n.9, p.1929-36, 2009. http://dx.doi.org/10.1590/S0102$\underline{311 X 2009000900007}$

PINHEIRO, R.S., et al. Gênero, morbidade, acesso e utilização de serviços de saúde no Brasil. Ciênc Saúde Coletiva, v.7, n.4, p.687-707, 2002.

http://dx.doi.org/10.1590/S1413-

$\underline{81232002000400007}$

SÁ, M.R.C.; GOMES, R. A promoção da saúde da criança: a participação da Fisioterapia. Cien Saude Colet., v.18, n.4, p.1079-88, 2013.

http://dx.doi.org/10.1590/S1413-

$\underline{81232013000400021}$

SATO, T.O., et al. Condições de vida e de saúde das famílias adscritas a Unidades de Saúde da Família. O Mundo da Saúde, v.39, n.1, p.74-83, 2015. http://DOI: $\underline{10.15343 / 0104-7809.201539017483}$ fisioterapia por um serviço de Atenção Primária à Saúde, 2012. Epidemiol. Serv. Saúde, Brasília, v. 24, n. 1, p. 123-133, 2015. http://dx.doi.org/10.5123/S1679$\underline{49742015000100014}$

SILVA, P.H.B.; LIMA, K.A.; LEROY, P.L.A. Perfil epidemiológico dos pacientes assistidos na clínica de fisioterapia traumatoortopédica da prefeitura de Hidrolândia Goiás. Movimenta, v.6, n.3, p.520-9, 2013. http://www.nee.ueg.br/seer/index.php/movim enta/article/viewFile/676/519

SIQUEIRA, F.V.; FACCHINI, L.A.; HALLAL, P.C. Epidemiology of physiotherapy utilization among adults and elderly. Rev Saude Publica, v.39, n.4, p.6638, 2005. http://dx.doi.org/10.1590/S0034$\underline{89102005000400022}$

TOSCANO, J.J.O.; EGYPTO, E.P. A influência do sedentarismo na prevalência de lombalgia. Rev Bras Med Esporte, v.7, n.4, p.132-137, 2001.

http://dx.doi.org/10.1590/S1517$\underline{86922001000400004}$

VASCONCELOS, M.K.P.; FAGUNDES, M.B.; GIUSTI, P.H. Resgate da fisioterapia ambulatorial realizada pelo sistema único de saúde desde a municipalização plena em Pelotas/RS. Bol Saude, v.1, n.2, p.35-40, 2007.

YAMAMOTO, F.I. Acidente vascular cerebral isquêmico em adultos jovens: considerações etiológicas. Arq Neuropsiquiatr., v.70, n.6, p.462-6, 2012. http://dx.doi.org/10.1590/S0004$\underline{282 X 2012000600014}$

SILVA, Guilherme Grivicich da; SIRENA, Sergio Antonio. Perfil de encaminhamentos a 